\title{
Optical bistability analysis inside a two-bus ring resonator.
}

\author{
Gino Priem, Pieter Dumon, Wim Bogaerts, Dries Van Thourhout, Geert Morthier, and Roel Baets \\ Photonics Research Group, Dept. of Information Technology, Ghent University - IMEC, Belgium \\ Gino.Priem@intec.UGent.be
}

\begin{abstract}
We demonstrate experimentally optical bistability inside a two-bus ring resonator fabricated in Silicon-on-Insulator with a power of only $680 \mu \mathrm{W}$. We have analysed the different contributions leading to this bistable behaviour and show that the dominant nonlinear effects are free carrier based.
\end{abstract}

\section{INTRODUCTION}

Photonic wire structures fabricated in high contrast systems such as Silicon-on-Insulator (SOI) allow strong transverse confinement of light within submicron dimensions. This optical confinement can even be further enhanced in the longitudinal direction by using resonant structures such as ring resonators, in which the propagation of the light pulse is slowed down.

Such structures with high confinement are of great interest for nonlinear optics, as they allow nonlinear effects to become applicable with only limited power levels. Nonlinear experiments in Silicon systems have already been performed by several groups for different types of resonant devices: singlebus (all-pass) ring resonators [1], $\mathrm{PhC}$ microresonators [2], [3].

We present nonlinear bistability, for the first time to our knowledge, inside a two-bus ring resonator with a power of only $680 \mu \mathrm{W}$. This is the lowest reported value for any ring resonator type so far.

\section{RESONATOR STRUCTURE.}

Resonator structures were created on $200 \mathrm{~mm}$ SOI wafers with a thickness of the Silicon layer of $220 \mathrm{~nm}$ and a burried oxide of $1 \mu \mathrm{m}$. A deep UV lithography stepper with a $248 \mathrm{~nm}$ illumination wavelength is used to define the patterns in the resist. A dry etching process then transfers the patterns into the Silicon layer. A detailed overview of the processing steps can be found in [4]. The processes are in se CMOS processes, characterized and adapted for the fabrication of photonic circuits, which enforces quite different technological boundary conditions. An example of an obtained resonator structure is shown in Figure 1.

The resonator structure measured in this paper has a radius of $4 \mu \mathrm{m}$ and wire width of approximately $540 \mathrm{~nm}$.

\section{MeAsurements}

To characterize the resonator structure, we used a tunable laser together with an Erbium-Doped fiber amplifier (EDFA) and variable attenuator, and detector. Coupling into and from the sample occurs by means of grating couplers optimized for

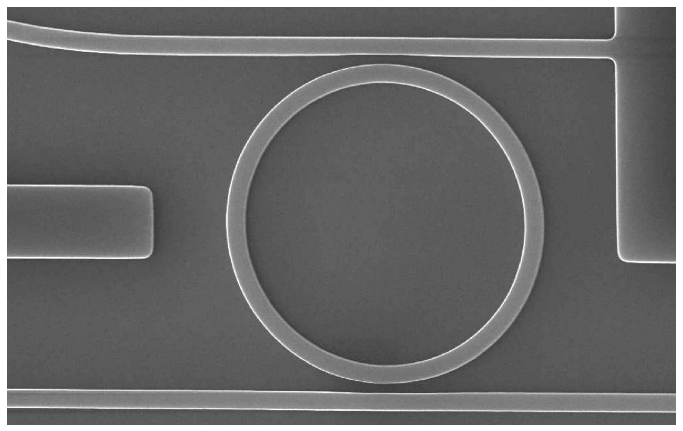

Fig. 1. Example of a ring resonator structure fabricated through deep UV lithography.

standard single-mode fiber [5]. These couplers have a $-7.5 d B$ maximum transmission and a $60 \mathrm{~nm} 3 \mathrm{~dB}$ bandwidth.

The obtained linear bandwidth of the ring resonator was $0.03 \mathrm{~nm}$, centered around the resonance wavelength $1556.75 \mathrm{~nm}$ and with a peak transmission of about $12 \%$. Measurements were done for in-wire input powers of 170, $340,680,1360$ and $2720 \mu \mathrm{W}$. The obtained nonlinear results are shown in Figure 2

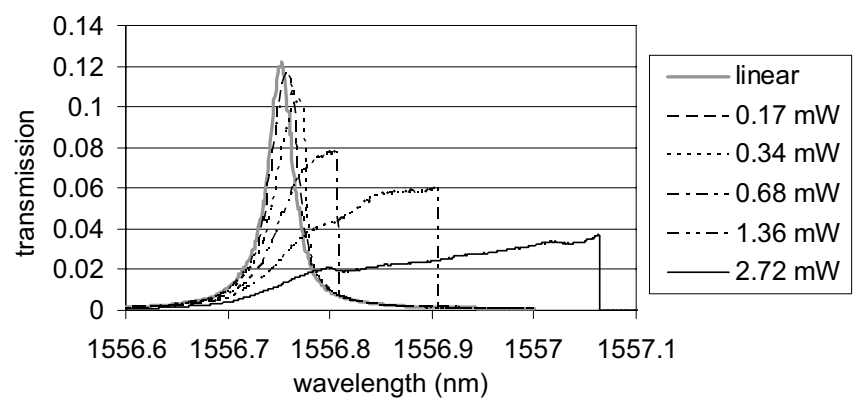

Fig. 2. Nonlinear transmission measurements of the ring resonator drop port for different input powers.

The strong step-like transmission changes obtained for 680,1360 and $2720 \mu W$ correspond to optical bistable behaviour. Due to the fact that the wavelength sweeping of the tunable laser occurs with interruption of the laser signal, only the lower arm of the bistability was obtained by the measurements. 


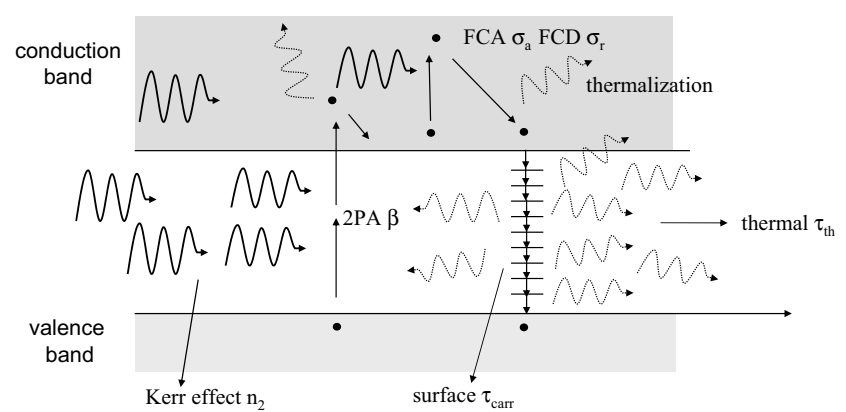

Fig. 3. Competing nonlinear effects in the Silicon material system for wavelengths around half the band gap.

\section{INTERPRETATION}

The complete nonlinear picture inside a structure such as a ring resonator is very complex: multiple nonlinear effects are competing with each other, which are all interdependent [2]. This is schematically presented in Figure 3.

When light enters in the Silicon material - which has a band gap close to twice the photon energy -, it can be absorbed through two-photon absorption (TPA) and give rise to a change in the refractive index effect proportional to the light intensity (Kerr effect). These free carriers that are excited through the TPA process, can give rise to additional absorption (Free Carrier Absorption, FCA) and an associated index change (Free Carrier Dispersion, FCD), which is proportional to the squared optical intensity. After a while, these carriers will recombine and in the case of submicron structures such as photonic wires, this is mainly due to surface recombination. This interband relaxation together with the intraband relaxation effects from carriers created due to TPA and FCA will meanly lead to phonon creation, which results in heating of the structure and gives rise to thermal expansion and a thermal refractive index change (consisting of linear, quadratic and cubic contributions in terms of the optical intensity). Due to conduction and convection, the structure finally cools down to a steady-state temperature.

The strength of these different refractive index effects was derived as a function of the optical power by fitting the different intensity dependencies mentioned above to the data obtained in Figure 2. This is shown in Figure 4.

As can be seen, the main contributions are caused by free carrier effects (i.e. FCD and thermalization of the FCA process). As both mechanisms are opposite in sign, they partially cancel out each other.

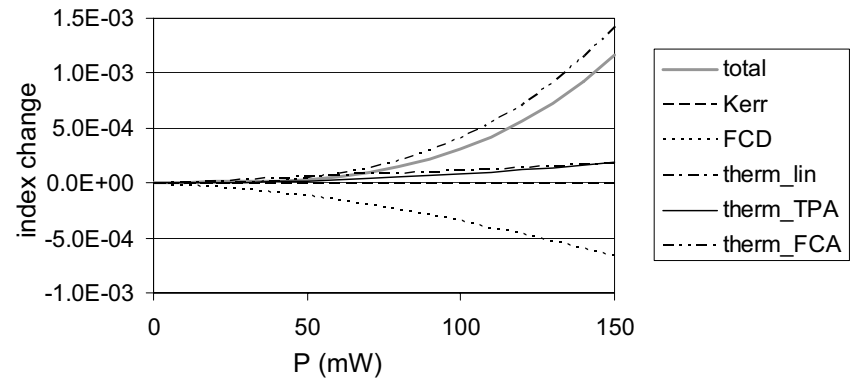

Fig. 4. Derived refractive index change for the different mechanisms, shown in Figure 3, as a function of power.

Additional thermal contributions (linear and TPA) further enhance the nonlinear refractive index change. The contribution of the Kerr-nonlinear effect on the other hand is negligible. These conclusions are in agreement with [2].

\section{Conclusions}

Nonlinear measurements of a two-port ring resonator structure fabricated in SOI have been demonstrated. Optical bistability has been observed for an input power of only $680 \mu \mathrm{W}$. The different refractive index contributions which lead to this bistable condition have been investigated and it is shown that that dominant effects were all free carrier related.

\section{ACKNOWLEDGMENT}

This work was supported by the EU through the Network of Excellence ePIXnet. Part of this work has been performed in the context of the Belgian IAP PHOTON Network (IAP $\mathrm{V} / 18)$.

Gino Priem acknowledges the Flemish Fund for Scientific Research (FWO-Vlaanderen) for financial support. Pieter Dumon thanks the Institute for the Promotion of Innovation through Science and Technology in Flanders (IWTVlaanderen) for a scholarship.

\section{REFERENCES}

[1] V. Almeida et al., "Optical bistability on a silicon chip," Opt. Lett., vol. 29, no. 20, pp. 2387-2389, 2004.

[2] P. Barclay et al., "Nonlinear response of silicon photonic crystal microresonators excited via an integrated waveguide and fiber taper," Opt. Expr., vol. 13, no. 3, pp. 801-820, 2005.

[3] M. Notomi et al., "Optical bistable switching action of si high-q photoniccrystal nanocavities,” Opt. Expr., vol. 13, no. 7, pp. 2678-2687, 2005.

[4] W. Bogaerts et al., "Nanophotonic waveguides in silicon-on-insulator fabricated with cmos technology," J. Lightwave Technol., vol. 23, no. 1, pp. 401-412, January 2005.

[5] D. Taillaert et al., "An out-of-plane grating coupler for efficient buttcoupling between compact planar waveguides and single-mode fibers," IEEE J. Quantum Electron., vol. 38, no. 7, pp. 949-955, 2002. 